\title{
Strategy-proof voting for single issues and cabinets
}

Citation for published version (APA):

Maus, S., Peters, H. J. M., \& Storcken, A. J. A. (2003). Strategy-proof voting for single issues and cabinets. METEOR, Maastricht University School of Business and Economics. METEOR Research Memorandum No. 029 https://doi.org/10.26481/umamet.2003029

Document status and date:

Published: 01/01/2003

DOI:

10.26481/umamet.2003029

Document Version:

Publisher's PDF, also known as Version of record

\section{Please check the document version of this publication:}

- A submitted manuscript is the version of the article upon submission and before peer-review. There can be important differences between the submitted version and the official published version of record.

People interested in the research are advised to contact the author for the final version of the publication, or visit the DOI to the publisher's website.

- The final author version and the galley proof are versions of the publication after peer review.

- The final published version features the final layout of the paper including the volume, issue and page numbers.

Link to publication

\footnotetext{
General rights rights.

- You may freely distribute the URL identifying the publication in the public portal. please follow below link for the End User Agreement:

www.umlib.nl/taverne-license

Take down policy

If you believe that this document breaches copyright please contact us at:

repository@maastrichtuniversity.nl

providing details and we will investigate your claim.
}

Copyright and moral rights for the publications made accessible in the public portal are retained by the authors and/or other copyright owners and it is a condition of accessing publications that users recognise and abide by the legal requirements associated with these

- Users may download and print one copy of any publication from the public portal for the purpose of private study or research.

- You may not further distribute the material or use it for any profit-making activity or commercial gain

If the publication is distributed under the terms of Article $25 \mathrm{fa}$ of the Dutch Copyright Act, indicated by the "Taverne" license above, 


\title{
Strategy-proof voting for single issues and cabinets
}

\author{
Stefan Maus* Hans Peters* Ton Storcken*
}

June 2003

\begin{abstract}
In a model with a continuum of voters with symmetric single-peaked preferences on the one-dimensional unit interval (representing the political spectrum) a voting rule assigns to each profile of votes a point in the interval. We characterize all voting rules that are strategy-proof, anonymous, Pareto optimal, and which satisfy a weak form of continuity. This result paves the way for studying cabinet formation rules. A cabinet is an interval which has obtained sufficiently many votes. The main result on cabinet formation is a characterization of all cabinet formation rules that are strategy-proof with respect to the endpoints of the cabinet, anonymous, Pareto optimal, and continuous.
\end{abstract}

Keywords: Strategy-proofness, voting rules, cabinet formation

Journal of Economic Literature Classification Numbers: D72, D71

*Department of Quantitive Economics, University of Maastricht, P.O. Box 616, 6200 MD Maastricht, The Netherlands. Telephone: +31-43-3883834. Telefax: +31-43-3884874. E-mail: s.maus@ke.unimaas.nl, h.peters@ke.unimaas.nl, t.storcken@ke.unimaas.nl. 


\section{Introduction}

In multiparty democracies, national elections typically have two objectives. The first objective is the choice of a new parliament as a direct representation of the voters' preferences. The second, indirect objective is the formation of a new cabinet by the newly chosen party representatives. The procedure for cabinet formation, however, is usually not very formal and is therefore often obscured by haggling between parties, driven by motives that can be quite distant from purely political points of view. Moreover, the fact that direct voting for parliament is used indirectly for cabinet formation may result in strategic manipulation by voters, possibly reinforced by the opaqueness of the cabinet formation procedure. Typically, for instance, voters tend to vote for parties in the center of the political spectrum in order to ensure that the resulting cabinet will be closer to their true - more left or right-political preferences, since parties away from the center usually have a smaller chance of participating in the new government.

In this paper we study procedures for cabinet formation that are both transparent - since they will be well-defined rules - and less vulnerable to strategic voting. As strategic voting may result in stronger center parties it may facilitate the formation of a cabinet $^{1}$, but it has the undesirable consequence of resulting in a parliament that does not properly reflect the preferences of the voters and therefore undermines the ideal of true proportional representation.

We assume that voters form a continuum - technically, an atomless measure space - as a best approximation of a very large set of voters. This assumption makes it also possible to endow single voters with zero influence, as is the case in reality. Strategic manipulation will be avoided by imposing a strategy-proofness condition, meaning that sets of voters of positive measure are not able to bend the formation of a cabinet in their preferred direction by voting differently from their preferences. Note that in our model only coalitions of voters can potentially manipulate, but this does not necessarily imply that voters actually convene to coordinate their voting behavior. Although single voters have zero influence, they may nevertheless derive utility

\footnotetext{
${ }^{1}$ Nevertheless, in the last Dutch elections for Parliament in January 2003, clearly the Social Democratic Party (PvdA) came out very strong as many left-oriented people voted for it in order to decrease the probability of a conservative cabinet, but in spite of this it did not make it to the new cabinet established in May 2003.
} 
from voting and, thus, may also want to vote strategically, possibly resulting in strategic behavior of groups of equally-minded voters.

Voters are assumed to have single-peaked symmetric preferences on the political spectrum, modeled as the interval $[0,1]$. Thus, a voter's preference is completely determined by its peak or ideal point in this interval. Preference decreases if we move away from this point, in either direction ${ }^{2}$.

Before we study cabinet formation we first consider single-issue voting. This is not only of interest by itself, but in particular paves the way for the subsequent models of cabinet formation. A voting rule assigns a point in $[0,1]$ to any profile of votes (e.g., tax level, spending on roads or on public transportation, etc.). We derive a characterization of the complete class of voting rules that satisfy the conditions of strategy-proofness, anonymity, Pareto optimality, and a weak form of continuity. This result can be seen as the extension of Moulin (1980) from a finite number of agents to a continuum.

In a model of cabinet formation that would be close to reality, we could assume the existence of $n$ political parties, located at fixed points in the political spectrum ${ }^{3}$. An election would result in a distribution of votes over these parties, and a cabinet could be naturally defined as a connected interval of parties such that the total number of votes exceeds a certain number (usually 50\%). Since we do not want to deal with the peculiarities associated with such a discrete approach, we will simply assume that voters can still vote for every point in the unit interval. A cabinet will be defined as a closed subinterval of the unit interval that has sufficient weight in the distribution of votes resulting from the election. So a cabinet in this sense reflects the positions that should be occupied by the political parties in the new government resulting from an election, rather than the concrete parties. The rules that we derive can nevertheless be used for the more realistic discrete model - they can be used to 'compute' the cabinet if we fix the positions of the existing political parties on the interval-but they are derived on the basis of conditions imposed in this more abstract continuous model.

Next, we extend the voters' preference relations to cabinets, i.e., closed intervals. Ideally, such a preference would depend not only on the location of the interval, that is, of its endpoints, but also on the distribution of votes over this interval. This, however, seems too much to ask for in the pres-

\footnotetext{
${ }^{2}$ See Section 5 for a brief discussion on extension to more dimensions.

${ }^{3}$ We do not consider strategic 'location' behavior by political parties in this paper.
} 
ence of nonmanipulability since, as usual, this condition necessarily implies a relatively strong insensitivity with respect to changes in preferences and therefore changes in the distribution of votes in an election ${ }^{4}$. Instead, we proceed in two steps. First, we assume that voters' preferences for a cabinet are determined by their preferences for a 'fixed' point in the cabinet. A natural choice for this could be the middle point of the cabinet but our result here is valid for any fixed convex combination of the endpoints of the cabinet. This result is based on our characterization of single-issue voting rules and characterizes all so-called cabinet formation rules that minimal, anonymous, and continuous, strategy-proof and Pareto optimal with respect to a fixed convex combination of the endpoints. The first mentioned condition says that there should not be a cabinet which is smaller on the left as well as on the right side and that still has the required majority of votes. Second, and this is the main result of the paper, we characterize all cabinet formation rules that are Pareto optimal in a strong sense (the cabinet is a subset of the convex hull of the support of the election distribution), continuous, anonymous, and strategy-proof with respect to both its endpoints. It will also be argued that these rules are strategy-proof with respect to a reasonable partial ordering (preference) on intervals (cabinets) - which is the most that can be expected since an unambiguously convincing extension of preferences to intervals does not exist. Moreover, these rules can be chosen in such a way that the resulting cabinets are close to minimal.

The literature on political coalition or cabinet formation includes both theoretical and empirical work and is so extensive that we do not even attempt to discuss it in any detail. For a recent survey, see De Vries (1999). Here we just mention a few early references closely related in spirit to our approach. Riker (1962) discusses the so called 'size principle' (Chs. 2-4) which implies that coalitions should not represent more parliamentary seats than necessary for the required majority, and this is consistent with the (close to) minimality condition that we impose on cabinets. Our main argument for a minimal cabinet is not so much that having additional members results in a decrease of per capita 'payoff' (as in Riker, 1962) but rather that in smaller cabinets it may be easier for the involved parties to compromise. Axelrod (1970) — see also De Swaan (1973) — argues that cabinets with minimal potential conflict of interest are likely to be formed, and this is reflected by

\footnotetext{
${ }^{4}$ This is an 'educated guess': we do not derive a formal impossibility result.
} 
our definition of a cabinet as an interval within the political spectrum. Our approach is uni-dimensional: in the concluding section we briefly discuss its potential extension to more dimensions and to a spatial voting model.

The organization of the paper is as follows. After the preliminaries in Section 2 we study single-issue voting rules in Section 3 and cabinet formation rules in Section 4. Section 5 concludes.

\section{Preliminaries}

Let $(\Omega, \Sigma, \lambda)$ be a nonatomic measure space. Every $t \in \Omega$ is a voter and every element $S$ of the $\sigma$-field $\Sigma$ is a coalition. The nonnegative number $\lambda(S)$ is interpreted as the size of $S$. We assume that $\Omega$ has positive measure and normalize its size to one: $\lambda(\Omega)=1$.

The set of alternatives is the real interval $A=[0,1]$. For a voter $t \in \Omega$, a single-peaked preference on $A$ is a complete and transitive binary relation $R(t)$, with asymmetric and symmetric parts denoted by $P(t)$ and $I(t)$ respectively, that has a peak $p(R(t)) \in A$, i.e., an alternative satisfying $p(R(t)) P(t) x P(t) y$ whenever $p(R(t))>x>y$ or $p(R(t))<x<y$. In this paper we consider only single-peaked preferences $R(t)$ that are symmetric around their peaks, i.e., if $x-p(R(t))=p(R(t))-y$ then $x I(t) y$. Such preferences are completely determined by their peaks, and we identify a symmetric preference $R(t)$ with its peak $p(R(t)) \in A$.

A profile (of preferences) is a measurable function $R: \Omega \rightarrow A$, where we take the Borel $\sigma$-field on $A$. The set of all profiles is denoted by $\rho$. Every $R \in \rho$ induces a (probability) measure $\lambda_{R}:=\lambda \circ R^{-1}$ on $A$. This measure $\lambda_{R}$ represents the distribution of votes resulting from an election where every voter $t \in \Omega$ votes according to $R(t)$.

Let $J$ be some ordered index set. A collection of profiles $\left(R_{j}\right)_{j \in J}$ converges to a profile $R$ if $\left(\lambda\left(\left\{t \in \Omega \mid R_{j}(t) \neq R(t)\right\}\right)\right)_{j \in J}$ converges to 0 .

\section{Voting for a single issue}

A (voting) rule is a function that assigns an alternative to each $R \in \rho$, i.e., a function $\varphi: \rho \rightarrow A$.

A rule $\varphi$ is continuous if $\left(\varphi\left(R_{j}\right)\right)_{j \in J}$ converges to $\varphi(R)$ whenever the 
collection of profiles $\left(R_{j}\right)_{j \in J}$ (where $J$ is an ordered index set) converges to $R \in \rho$. Observe that continuity of a rule $\varphi$ implies that coalitions of size zero and in particular single voters are powerless: that is, $\varphi(R)=\varphi(\widetilde{R})$ whenever $\lambda(\{t \in \Omega \mid R(t) \neq \widetilde{R}(t)\})=0$. Also observe that this is a very weak continuity condition: it does not imply anything if each voter's preferences in two different profiles are very close.

A rule $\varphi$ is Pareto optimal if $\varphi(R)$ is an element of the convex hull of the support of $\lambda_{R}$ for every $R \in \rho$. Note that this is always a closed interval in $A$. Also note that this notion of Pareto optimality is equivalent to the basic condition that there is no alternative that is better for all voters in a coalition of positive size without being worse for all voters in another coalition of positive size.

A rule $\varphi$ is manipulable by a coalition $S$ at a profile $R \in \rho$ if there is a profile $\widetilde{R} \in \rho$ with $\widetilde{R}(t)=R(t)$ for all $t \in \Omega \backslash S$ such that $\varphi(\widetilde{R}) P(t) \varphi(R)$ for all $t \in S$. A rule $\varphi$ is strategy-proof if it is not manipulable by any coalition $S$ at any profile $R$.

A rule $\varphi$ is anonymous if for all $R, \widetilde{R} \in \rho$, if $\lambda_{R}=\lambda_{\widetilde{R}}$, then $\varphi(R)=\varphi(\widetilde{R})$.

The goal of this section is to characterize all rules that are continuous, Pareto optimal, anonymous, and strategy-proof. In this characterization, a central role is played by the family $F$ of all nondecreasing (i.e., weakly increasing) and continuous functions $f:[0,1] \rightarrow A$ with $f(0)=0$ and $f(1)=1$. Let $f \in F$. We define a rule $\varphi^{f}$ associated with $f$, as follows (see Figure 1 for an illustration).

Let $R \in \rho$. Consider the induced decumulative distribution $D^{R}$ defined by $D^{R}(x):=\lambda_{R}([x, 1])$ for all $x \in A$. Now

$$
\varphi^{f}(R):=\max \left\{x \in A \mid f\left(D^{R}(x)\right) \geq x\right\} .
$$

The rule $\varphi^{f}$ is well-defined since the set of which the maximum is taken is nonempty (it contains $x=0$ ), $f$ is continuous and $D^{R}$ is left-continuous.

The announced result is the following.

Theorem 3.1 A rule $\varphi$ is continuous, Pareto optimal, strategy-proof and anonymous if and only if there is an $f \in F$ such that $\varphi=\varphi^{f}$.

We first prove the if-direction of this theorem.

Proposition 3.2 Let $f \in F$. Then $\varphi^{f}$ is continuous, Pareto optimal, anonymous, and strategy-proof. 

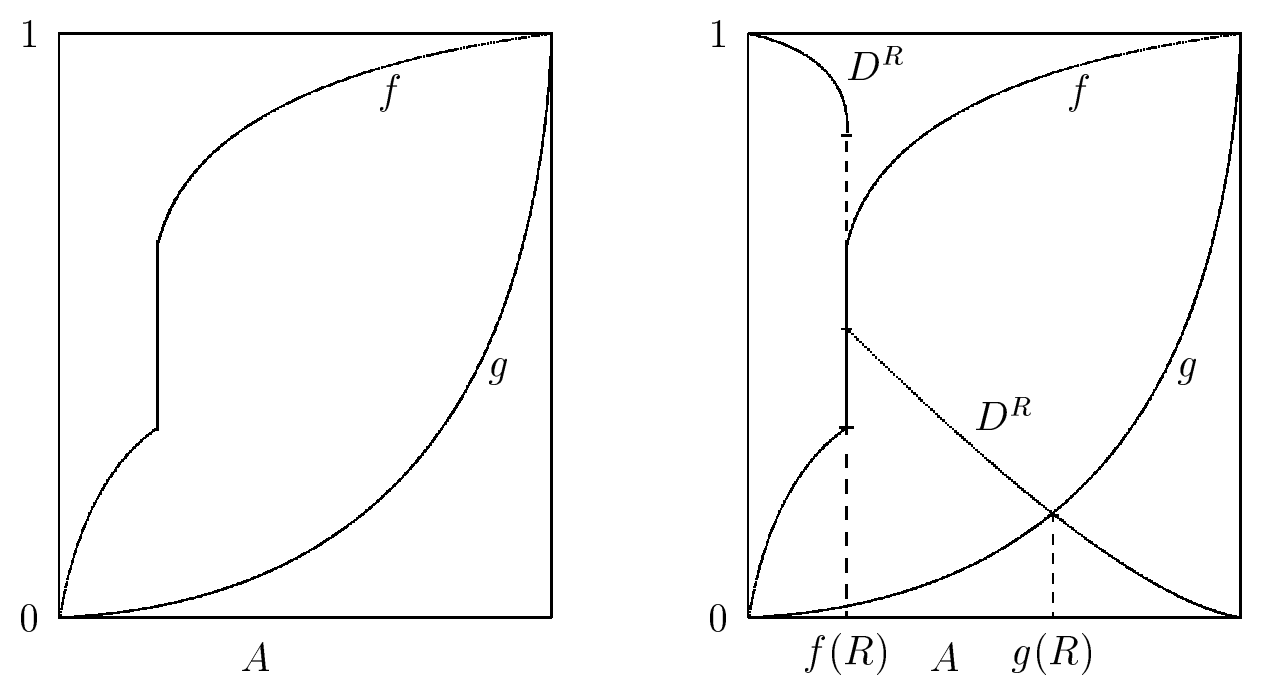

Figure 1: The left diagram shows two different functions $f, g \in F$. The domain of these functions is on the vertical axis, and the range is the set $A$ on the horizontal axis. The right diagram depicts a distribution $D^{R}$ and the associated points $\varphi^{f}(R)$ and $\varphi^{g}(R)$.

Proof. For continuity, let $J$ be an ordered index set and let $\left(R_{j}\right)_{j \in J}$ be a collection of profiles converging to $R \in \rho$. Then $\left(\lambda_{R_{j}}(B)\right)_{j \in J}$ converges to $\lambda_{R}(B)$ for every Borel set $B \subseteq A$, so in particular, for every $x \in A,\left(D^{R_{j}}(x)\right)_{j \in J}$ converges to $D^{R}(x)$. By continuity of $f$ this implies that $(\max \{x \in A$ | $\left.\left.f\left(D^{R_{j}}(x)\right) \geq x\right\}\right)_{j \in J}$ converges to $\max \left\{x \in A \mid f\left(D^{R}(x)\right) \geq x\right\}$, hence $\left(\varphi^{f}\left(R^{j}\right)\right)_{j \in J}$ converges to $\varphi^{f}(R)$.

For Pareto optimality, let $R \in \rho$ and let $[a, b]$ be the convex hull of the support of $\lambda_{R}$. Since $D^{R}(a)=1$ we have $f\left(D^{R}(a)\right)=1 \geq a$, hence $\varphi^{f}(R) \geq a$. Since $D^{R}(b)=0$ we have $\varphi^{f}\left(D^{R}(x)\right)=0<x$ for every $x>b$, hence $\varphi^{f}(R) \leq b$.

For anonymity, if $R, R^{\prime}$ are profiles with $\lambda_{R}=\lambda_{R^{\prime}}$, then $D^{R}=D^{R^{\prime}}$, hence $\varphi^{f}(R)=\varphi^{f}\left(R^{\prime}\right)$.

Finally, we show strategy-proofness of $\varphi^{f}$. Let $R \in \rho$ and $S \in \Sigma$ and suppose that $\varphi^{f}$ is manipulable by $S$ at $R$. Then there is a profile $\widetilde{R} \in \rho$ with $\widetilde{R}(t)=R(t)$ for all $t \in \Omega \backslash S$ and $\varphi^{f}(\widetilde{R}) P(t) \varphi^{f}(R)$ for all $t \in S$. Because of single-peakedness we have either $R(t)<\varphi^{f}(R)$ for all $t \in S$ or $R(t)>\varphi^{f}(R)$ for all $t \in S$. Without loss of generality assume $R(t)<\varphi^{f}(R)$ for all $t \in S$, the other case is similar. Then, for all $x \geq \varphi^{f}(R)$, we have $D^{\widetilde{R}}(x) \geq D^{R}(x)$ 
since $\widetilde{R}(t)=R(t)$ for all $t \in \Omega \backslash S$. This implies

$$
\varphi^{f}(R) \leq f\left(D^{R}\left(\varphi^{f}(R)\right)\right) \leq f\left(D^{\widetilde{R}}\left(\varphi^{f}(R)\right)\right),
$$

hence $\varphi^{f}(\widetilde{R}) \geq \varphi^{f}(R)$. So $\varphi^{f}(R) R(t) \varphi^{f}(\widetilde{R})$ for all $t \in S$, a contradiction.

The following lemma is crucial for the proof of the only-if direction of Theorem 3.1. It shows the analogon of the usual 'uncompromisingness' condition associated with strategy-proofness (cf. Border and Jordan, 1983).

Lemma 3.3 Let the rule $\varphi$ be continuous, anonymous, and strategy-proof. Let $R, R^{\prime} \in \rho$. Then:

(i) If $R^{\prime}(t)=R(t)$ for all $t \in \Omega$ with $R(t) \geq \varphi(R)$ and $R^{\prime}(t)<\varphi(R)$ for all $t \in \Omega$ with $R(t)<\varphi(R)$, then $\varphi\left(R^{\prime}\right)=\varphi(R)$.

(ii) If $R^{\prime}(t)=R(t)$ for all $t \in \Omega$ with $R(t) \leq \varphi(R)$ and $R^{\prime}(t)>\varphi(R)$ for all $t \in \Omega$ with $R(t)>\varphi(R)$, then $\varphi\left(R^{\prime}\right)=\varphi(R)$.

Proof. We only prove (i), (ii) is analogous. To prove (i), it is sufficient to prove that $\varphi\left(R^{\prime}\right)=\varphi(R)$ if $R(t)=R^{\prime}(t)$ for all $t \in \Omega$ with $R(t) \geq \varphi(R)$, and $R^{\prime}(t)=0$ for all other $t \in \Omega$.

Let $\gamma:=1-D^{R}(\varphi(R))$, i.e., $\gamma$ is the size of the coalition of voters that vote strictly left from $\varphi(R)$. For every $0 \leq \alpha \leq \gamma$ let $x^{\alpha}$ be the maximal point of $A$ with $D^{R}\left(x^{\alpha}\right) \geq 1-\alpha$ (hence $D^{R}(y)<1-\alpha$ for all $y>x_{\alpha}$ ), and choose $S^{\alpha} \subseteq R^{-1}\left(x^{\alpha}\right)$ such that $\lambda\left(R^{-1}\left(\left[0, x^{\alpha}\right)\right) \cup S^{\alpha}\right)=\alpha\left(S^{\alpha}\right.$ is possibly empty, in particular if $x^{\alpha}$ is not an atom of $\left.\lambda_{R}\right)$. Now define the profile $R^{\alpha}$ by

$$
R^{\alpha}(t):= \begin{cases}0 & \text { for all } t \in R^{-1}\left(\left[0, x^{\alpha}\right)\right) \cup S^{\alpha} \\ R(t) & \text { for all other } t \in \Omega .\end{cases}
$$

Then $R^{0}=R$, except possibly for a set of voters of size zero, and $R^{\gamma}=R^{\prime}$. Take $0 \leq \alpha<\gamma$, then $0 \leq x^{\alpha}<\varphi(R)$. We distinguish two cases:

(a) $\varphi\left(R^{\alpha}\right) \geq x^{\alpha}$. In this case, $\varphi\left(R^{\alpha}\right) \leq \varphi(R)$ since otherwise the coalition $\left(R^{\alpha}\right)^{-1}(0)$ could manipulate at $R^{\alpha}$ by inducing the profile $R$. If $\varphi\left(R^{\alpha}\right)<$ $\varphi(R)$, then the coalition $R^{-1}\left(\left[0, x^{\alpha}\right)\right) \cup S^{\alpha}$ can manipulate at $R$ by inducing the profile $R^{\alpha}$. We conclude that, in this case (a), $\varphi\left(R^{\alpha}\right)=\varphi(R)$.

(b) $\varphi\left(R^{\alpha}\right)<x^{\alpha}$. Then, by continuity of $\varphi$, there is an $\alpha^{\prime}$ with $0<\alpha^{\prime}<\alpha$ 
and $x^{\alpha^{\prime}} \leq x^{\alpha}<\varphi\left(R^{\alpha^{\prime}}\right)<\varphi(R)$. This, however, contradicts case (a) and therefore cannot occur.

Thus, for every $0 \leq \alpha<\gamma$, we have $\varphi\left(R^{\alpha}\right)=\varphi(R)$. The result now follows by using again the continuity of $\varphi$.

Proposition 3.4 Let the rule $\varphi$ be continuous, Pareto optimal, anonymous, and strategy-proof. Then there is an $f \in F$ such that $\varphi=\varphi^{f}$.

Proof. We first construct the desired function $f$. For every $\alpha \in[0,1]$, let $R_{\alpha} \in \rho$ be a profile with $\lambda_{R_{\alpha}}(\{1\})=\alpha$ and $\lambda_{R_{\alpha}}(\{0\})=1-\alpha$. Define

$$
f(\alpha):=\varphi\left(R_{\alpha}\right) \text { for every } \alpha \in[0,1] .
$$

This is a correct definition in view of the anonymity of $\varphi$. By Pareto optimality, $f(0)=0$ and $f(1)=1$. Continuity of $\varphi$ implies continuity of $f$. To show that $f$ is nondecreasing suppose, to the contrary, that $0 \leq \beta<\alpha \leq 1$ but $f(\alpha)<f(\beta)$. Let $S$ and $T$ be disjoint coalitions with $\lambda(S)=\beta$ and $\lambda(T)=\alpha-\beta$. Consider the profile $R_{\beta}$ with $R_{\beta}(t)=1$ for all $t \in S$ and $R_{\beta}(t)=0$ for all $t \in \Omega \backslash S$, and the profile $R_{\alpha}$ with $R_{\alpha}(t)=1$ for all $t \in S \cup T$ and $R_{\alpha}(t)=0$ for all $t \in \Omega \backslash(S \cup T)$. Since $f(\alpha)<f(\beta)$, hence $\varphi\left(R_{\alpha}\right)<\varphi\left(R_{\beta}\right)$, coalition $T$ can manipulate $\varphi$ at $R_{\alpha}$ by voting for 0 , so by inducing the profile $R_{\beta}$. This contradicts strategy-proofness of $\varphi$. Thus, $f$ is nondecreasing, so $f \in F$.

It follows by the construction of $f$ that

$$
\varphi\left(R_{\alpha}\right)=\varphi^{f}\left(R_{\alpha}\right) \text { for every } \alpha \in[0,1] .
$$

Now let $R \in \rho$ be an arbitrary profile and denote $z:=\varphi(R) \in A$. We prove that $\varphi^{f}(R)=z$.

First consider the profile $R^{\prime}$ defined by

$$
R^{\prime}(t):= \begin{cases}0 & \text { if } R(t)<z \\ z & \text { if } R(t)=z \\ 1 & \text { if } R(t)>z\end{cases}
$$

By Lemma 3.3 we have $\varphi\left(R^{\prime}\right)=z$. Let $S=R^{-1}(z)$, and define the profiles $R^{0}$ and $R^{1}$ by

$$
R^{0}(t):= \begin{cases}0 & \text { if } R(t)<z \text { or } t \in S \\ 1 & \text { if } R(t)>z\end{cases}
$$


and

$$
R^{1}(t):= \begin{cases}0 & \text { if } R(t)<z \\ 1 & \text { if } R(t)>z \text { or } t \in S .\end{cases}
$$

Then by strategy-proofness we have $\varphi\left(R^{0}\right) \leq z \leq \varphi\left(R^{1}\right)$. Therefore, by continuity of $\varphi$ there is a partition of $S$ in sets $S^{0}$ and $S^{1}$ such that $z=\varphi\left(R_{\alpha}\right)$, where $\alpha=\lambda\left(S^{1} \cup\left(R^{\prime}\right)^{-1}(1)\right)$ and $1-\alpha=\lambda\left(S^{0} \cup\left(R^{\prime}\right)^{-1}(0)\right)$. Note that, by construction of $R_{\alpha}$, we have

$$
D^{R}(x) \geq D^{R_{\alpha}}(x) \text { for all } x \in A \text { with } x \leq z
$$

and

$$
D^{R}(x) \leq D^{R_{\alpha}}(x) \text { for all } x \in A \text { with } x>z .
$$

Since $z=\varphi\left(R_{\alpha}\right)$ we have by $(2)$ that $z=\varphi^{f}\left(R_{\alpha}\right)$ and thus, by definition,

$$
z=\max \left\{x \in A \mid f\left(D^{R_{\alpha}}(x)\right) \geq x\right\} .
$$

By (5) and (3), we have

$$
\max \left\{x \in A \mid f\left(D^{R}(x)\right) \geq x\right\} \geq z .
$$

Suppose the inequality in (6) were strict. Then there would be an $\widetilde{x}>z$ with $f\left(D^{R}(\widetilde{x})\right) \geq \widetilde{x}$. Hence by $(4), f\left(D^{R_{\alpha}}(\widetilde{x})\right) \geq f\left(D^{R}(\widetilde{x})\right) \geq \widetilde{x}$, contradicting (5). So we have an equality in (6), implying that $z=\varphi^{f}(R)$.

Theorem 3.1 now follows from Propositions 3.2 and 3.4.

For finitely many voters, Moulin (1980) characterizes all rules (on the real line, but that difference is not essential) that are anonymous, Pareto optimal, strategy-proof, and peaks-only. The last condition means that the rule depends only on the peaks of the preferences: in Moulin (1980) the domain also contains all nonsymmetric single-peaked preferences. Ching (1997) and Ehlers et al. (2002) show that the peaks-onliness condition can be dropped. In our case, we need continuity (see Example 3.5 below) since we have excluded nonsymmetric preferences from the domain. The role of a set of 'fixed ballots' in Moulin (1980), which characterizes a particular rule, is played in our paper by a function $f \in F$.

We conclude this section by showing independence of the conditions in Theorem 3.1 by means of four examples. In particular, Remark 3.7 gives the exact consequences of dropping Pareto optimality in Theorem 3.1. 
Example 3.5 The following rule $\varphi$ satisfies all conditions in Theorem 3.1 except continuity. Let $R \in \rho$ and let $[a, b]$ be the convex hull of the support of $\lambda_{R}$. Then $\varphi(R):=b$ if $\lambda_{R}([(a+b) / 2, b]) \geq 1 / 2$, and $\varphi(R):=a$ otherwise.

Example 3.6 The rule assigning 0 to every profile of preferences satisfies all conditions in Theorem 3.1 except Pareto optimality.

Remark 3.7 It is not difficult to describe the class of rules satisfying all conditions in Theorem 3.1 except possibly Pareto optimality. Let $\widetilde{F}$ be the family of all nondecreasing and continuous functions $f:[0,1] \rightarrow A$ (hence, $F=\{f \in \widetilde{F} \mid f(0)=0, f(1)=1\})$. For $f \in \widetilde{F}$ define the rule $\varphi^{f}$ as in (1). These rules $\varphi^{f}(f \in \widetilde{F})$ are exactly the rules satisfying continuity, anonymity, and strategy-proofness. This can be verified by going over the proof of Theorem 3.1.

Example 3.8 The rule assigning the expected alternative, i.e., the point $\int_{A} x d \lambda_{R}$, to every profile $R \in \rho$, satisfies all conditions in Theorem 3.1 except strategy-proofness.

Example 3.9 Fix a coalition $S \in \Sigma$ of positive size and fix $f \in F$. For a profile $R \in \rho$ define the decumulative distribution function $D_{S}^{R}: A \rightarrow[0,1]$ by

$$
D_{S}^{R}(x):=\frac{\lambda\left(R^{-1}([x, 1]) \cap S\right)}{\lambda(S)} \text { for every } x \in A .
$$

Let

$$
\varphi(R):=\max \left\{x \in A \mid f\left(D_{S}^{R}(x)\right) \geq x\right\} .
$$

This rule $\varphi$ satisfies all conditions in Theorem 3.1 except anonymity.

\section{Cabinet formation}

Let $p \in \mathbb{R}$ with $\frac{1}{2}<p \leq 1$ be fixed. The number $p$ expresses the fraction of total votes needed for a 'majority' cabinet. For a profile $R \in \rho$, a cabinet is an interval $[a, b] \subseteq A$ with $\lambda_{R}([a, b]) \geq p$. A cabinet formation rule $(\mathrm{CFR}) \Phi$ assigns to each profile $R \in \rho$ a cabinet.

A cabinet $[a, b]$ is minimal for a profile $R \in \rho$ if there is no cabinet $[c, d]$ for $R$ with $a<c \leq d<b$ and $\lambda_{R}([c, d]) \geq p$. Thus, a minimal cabinet cannot 
be shrunk on both the left and the right side without losing its majority. A CFR is minimal if it assigns a minimal cabinet to each profile.

In order to define further properties for a CFR we need to be more specific about the preferences of voters for different cabinets. As mentioned in the Introduction, ideally one might want these preferences to reflect not only the location of the cabinet but also the distribution of votes over the cabinet. For instance, if a voter's peak is to the left of a cabinet then, other things being equal, that voter would probably prefer more votes towards its left endpoint since the associated political party would have more power (more ministers) in the cabinet. This, however, seems hard to reconcile with strategy-proofness. We do not derive a formal impossibility result; indicative of the associated difficulties is for instance the voting rule in Example 3.8. Instead, we first assume that the preference of a voter for a specific cabinet is determined by a fixed convex combination of its endpoints - taking the middle point would be an obvious choice, for instance. Later, in our main result, we consider both endpoints.

Formally, let $\pi$ be a real number with $0 \leq \pi \leq 1$. For a cabinet $[a, b]$, denote by $m^{\pi}([a, b])$ the point $a+\pi(b-a)$. For a voter $t \in \Omega$ with preference $R(t) \cong p(R(t)) \in A$, we say that this voter (weakly) $m^{\pi}$-prefers cabinet $[a, b]$ to cabinet $[c, d]$ if he (weakly) prefers $m^{\pi}([a, b])$ to $m^{\pi}([c, d])$, i.e., if $\left|p(R(t))-m^{\pi}([a, b])\right| \leq\left|p(R(t))-m^{\pi}([c, d])\right|$. Observe that for $a=b$ and $c=d$ this is consistent with the preference $a R(t) b$. Therefore we can use the same notations for preferences and profiles as in the single issue case.

Let $J$ be an ordered index set. A CFR $\Phi$ is $m^{\pi}$-continuous if $\left(m^{\pi}\left(\Phi\left(R_{j}\right)\right)\right)_{j \in J}$ converges to $m^{\pi}(\Phi(R))$ whenever $\left(R_{j}\right)_{j \in J}$ converges to $R$.

A CFR $\Phi$ is anonymous if for all $R, \widetilde{R} \in \rho$, if $\lambda_{R}=\lambda_{\widetilde{R}}$, then $\Phi(R)=\Phi(\widetilde{R})$.

A CFR $\Phi$ is $m^{\pi}$-manipulable by a coalition $S$ at a profile $R \in \rho$ if there is a profile $\widetilde{R} \in \rho$ with $\widetilde{R}(t)=R(t)$ for all $t \in \Omega \backslash S$ such that $\Phi(\widetilde{R}) P(t) \Phi(R)$ for all $t \in S$. A CFR $\Phi$ is $m^{\pi}$-strategy-proof if it is not $m^{\pi}$-manipulable by any coalition $S$ at any profile $R$.

A CFR $\Phi$ is $m^{\pi}$-Pareto optimal if $m^{\pi}(\Phi(R))$ is in the convex hull of the support of $\lambda_{R}$ for every profile $R$.

Our purpose is to characterize all minimal cabinet formation rules that satisfy these four properties. To this end, we let $G^{\pi}$ denote the family of all nondecreasing continuous functions $g:[0,1] \rightarrow A$ with $g(0)=0, g(1-p)=$ $g(p)=\pi$, and $g(1)=1$. Observe that $G^{\pi} \subseteq F$.

Let $g \in G^{\pi}$. We define a $\mathrm{CFR} \Phi^{g}$ associated with $g$, as follows (see 


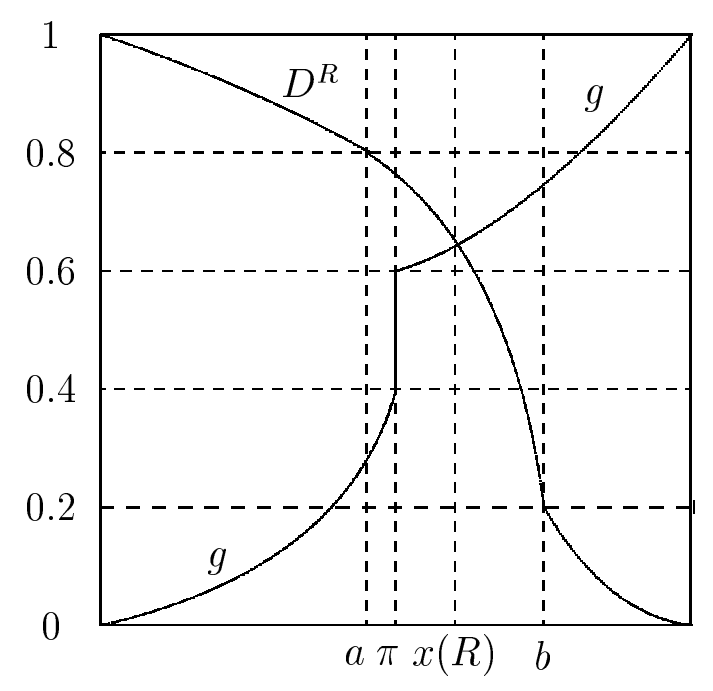

Figure 2: This diagram depicts a function $g \in G^{\pi}$, where $\pi=1 / 2$, with domain on the vertical axis, and a distribution $D^{R}$. The set of alternatives $A$ is on the horizontal axis. Furthermore, $p=0.6$, and the resulting cabinet is $[a, b]=[a(R), b(R)]$. The point $x(R)$ is the midpoint of $[a, b]$, and the weight of $[a, b]$ is equal to $p=0.6$.

Figure 2 for an illustration). For $R \in \rho$ let $D^{R}$ be the induced decumulative distribution as defined in Section 3. Also, let $D^{R}(x)^{+}:=\lambda_{R}((x, 1])$ for all $x \in A$. First define

$$
x(R):=\max \left\{x \in A \mid g\left(D^{R}(x)\right) \geq x\right\} .
$$

By the same arguments as used in Section 3, the point $x(R)$ is well-defined. We claim that there is a unique interval $[a(R), b(R)]$ with

(C1) $x(R)=a(R)+\pi(b(R)-a(R))$,

(C2) $D^{R}(a(R))-D^{R}(b(R))^{+} \geq p$, and

(C3) $[a(R), b(R)]$ is minimal for $R$.

To see this, first consider the case where $x:=x(R)<\pi$. The interval $[a, b]$ with $a=0$ and $b=x / \pi$ has $x=m^{\pi}([a, b])$. Take $\varepsilon>0$ with $x+\varepsilon<\pi$. If $D^{R}(x+\varepsilon)>1-p$ then $g\left(D^{R}(x+\varepsilon)\right) \geq \pi>x+\varepsilon$, a contradiction with the definition of $x=x(R)$. Hence, $D^{R}(x+\varepsilon) \leq 1-p$, so $D^{R}(b)^{+} \leq 1-p$ since 
$D^{R}$ is nonincreasing. Therefore $D^{R}(a)-D^{R}(b)^{+} \geq 1-(1-p)=p$, implying that $[a, b]$ satisfies (C.2). The case $x>\pi$ is analogous, and if $x=\pi$ consider the interval $A=[0,1]$. So in every case, an interval satisfying (C.1) and (C.2) exists. The existence of a minimal interval with these properties follows since $D^{R}$ is left-continuous and nonincreasing. Now, define $\Phi^{g}(R):=[a(R), b(R)]$.

The first result in this section is the following theorem, which for a given $\pi$ characterizes all minimal cabinet formation rules under conditions similar to those in Theorem 3.1 for voting rules.

Theorem 4.1 A CFR $\Phi$ is minimal, $m^{\pi}$-continuous, $m^{\pi}$-Pareto optimal, anonymous, and $m^{\pi}$-strategy-proof if and only if there is a $g \in G^{\pi}$ such that $\Phi=\Phi^{g}$.

The proof of this theorem is to a large extent analogous to the proof of Theorem 3.1. We start with the if-direction.

Proposition 4.2 Let $g \in G^{\pi}$. Then $\Phi^{g}$ is minimal, $m^{\pi}$-continuous, $m^{\pi}$ Pareto optimal, anonymous, and $m^{\pi}$-strategy-proof.

Proof. Minimality of $\Phi^{g}$ holds by definition. The other properties follow, basically, by Proposition 3.2, noting that $G^{\pi} \subseteq F$.

The proof of the following lemma is analogous to the proof of Lemma 3.3, and therefore omitted.

Lemma 4.3 Let the CFR $\Phi$ be $m^{\pi}$-continuous, anonymous, and $m^{\pi}$-strategyproof. Let $R, R^{\prime} \in \rho$. Then:

(i) If $R^{\prime}(t)=R(t)$ for all $t \in \Omega$ with $R(t) \geq m^{\pi}(\Phi(R))$ and $R^{\prime}(t)<$ $m^{\pi}(\Phi(R))$ for all $t \in \Omega$ with $R(t)<m^{\pi}(\Phi(R))$, then $m^{\pi}\left(\Phi\left(R^{\prime}\right)\right)=$ $m^{\pi}(\Phi(R))$.

(ii) If $R^{\prime}(t)=R(t)$ for all $t \in \Omega$ with $R(t) \leq m^{\pi}(\Phi(R))$ and $R^{\prime}(t)>$ $m^{\pi}(\Phi(R))$ for all $t \in \Omega$ with $R(t)>m^{\pi}(\Phi(R))$, then $m^{\pi}\left(\Phi\left(R^{\prime}\right)\right)=$ $m^{\pi}(\Phi(R))$.

The next proposition establishes the only-if direction of Theorem 4.1. 
Proposition 4.4 Let the CFR $\Phi$ be minimal, $m^{\pi}$-continuous, $m^{\pi}$-Pareto optimal, anonymous, and $m^{\pi}$-strategy-proof. Then there is a $g \in G^{\pi}$ such that $\Phi=\Phi^{g}$.

Proof. We first construct the desired function $g \in G^{\pi}$. Let the profiles $R_{\alpha}$ $(0 \leq \alpha \leq 1)$ be defined just as in the proof of Proposition 3.4. Denote $[a(\alpha), b(\alpha)]:=\Phi\left(R_{\alpha}\right)$ and let

$$
g(\alpha):=m^{\pi}([a(\alpha), b(\alpha)]) \text { for every } \alpha \in[0,1] .
$$

Then $g(0)=0$ and $g(1)=1$ by Pareto optimality of $\Phi$. For $\alpha<p$ we have $a(\alpha)=0$ by (C.2), hence $g(\alpha) \leq \pi$. For $\alpha>1-p$ we have $b(\alpha)=1$ by (C.2), hence $g(\alpha) \geq \pi$. Continuity of $g$ follows from $m^{\pi}$-continuity of $\Phi$, and by a proof similar as in Proposition 3.4, $m^{\pi}$-strategy-proofness of $\Phi$ implies that $g$ is nondecreasing. Hence, $g \in G^{\pi}$ and moreover, by construction, $\Phi\left(R_{\alpha}\right)=\Phi^{g}\left(R_{\alpha}\right)$ for all $\alpha \in[0,1]$.

Now let $R$ be an arbitrary profile. We want to prove $\Phi(R)=\Phi^{g}(R)$. By minimality of $\Phi$ and $\Phi^{g}$ it is sufficient to prove that $m^{\pi}(\Phi(R))=m^{\pi}\left(\Phi^{g}(R)\right)$. By using Lemma 4.3 instead of Lemma 3.3 this follows analogously to the last part of the proof of Proposition 3.4.

Theorem 4.1 follows from Propositions 4.2 and 4.4.

Call a CFR $\Phi$ Pareto optimal if $\Phi(R)$ is a subset of the convex hull of the support of $\lambda_{R}$, for every profile $R \in \rho$. The following example shows that, if voters have $m^{\pi}$-preferences with $0<\pi<1$, then a minimal, $m^{\pi}$-strategyproof, anonymous CFR cannot be Pareto optimal.

Example 4.5 Let $a \in A$ with $a<\frac{1}{2}$ and such that $m^{\pi}([a, 1])=a+\pi(1-a)>$ $\frac{1}{2}$ (this is possible since $\pi>0$ ). Consider a profile $R$ such that $\lambda_{R}$ distributes probability $p$ uniformly over the interval $\left[a, \frac{1}{2}\right]$ and puts weight $1-p$ on the point 1 . The minimal cabinets that can possibly be chosen by a Pareto optimal CFR are all cabinets of the form $[a, c]$ with $\frac{1}{2} \leq c \leq 1$; and all cabinets of the form $[c, 1]$ with $a \leq c \leq c^{\prime}$, where $a<c^{\prime}<\frac{1}{2}$ is chosen such that the interval $\left[c^{\prime}, 1\right]$ receives probability $p .^{5}$ Let $\Phi$ be a minimal, $m^{\pi}$-strategy-proof, and anonymous CFR.

\footnotetext{
${ }^{5}$ If we strengthen the minimality condition in the sense that there should be no proper subinterval with probability at least $p$, then the only minimal cabinets would be $\left[a, \frac{1}{2}\right]$ and $\left[c^{\prime}, 1\right]$. This does not change the example nor the claim it proves.
} 
First suppose that $\Phi(R)=[a, c]$ for some $\frac{1}{2} \leq c \leq 1$. Choose $a<b<$ $m^{\pi}(a, c)$ and consider the profile $R^{\prime}$ derived from $R$ by letting the voters in $R^{-1}([a, b])$ vote for $b$. By $m^{\pi}$-strategy-proofness, $m^{\pi}\left(\Phi\left(R^{\prime}\right)\right) \leq m^{\pi}(\Phi(R))=$ $m^{\pi}([a, c])$. Since $\Phi\left(R^{\prime}\right)$ has to have at least probability $p$, we conclude that it contains at least $\left[a, \frac{1}{2}\right]$. This violates Pareto optimality since the convex hull of the support of $\lambda_{R^{\prime}}$ is the interval $[b, 1]$.

Next, suppose that $\Phi(R)=[c, 1]$ for some $c$ with $a \leq c \leq c^{\prime}$. Then $m:=m^{\pi}(\Phi(R))>\frac{1}{2}$. Then $m<1$ since $\pi<1$, so we can choose an $\varepsilon>0$ such that $m-\varepsilon>\frac{1}{2}$ and $m+\varepsilon<1$. Consider the profile $\widetilde{R}$ derived from $R$ by letting the voters in $R^{-1}(1)$ vote for $m+\varepsilon$. Then by $m^{\pi}$-strategy-proofness, $m^{\pi}(\Phi(\widetilde{R}))=m$. In order to have probability at least $p$ on $\Phi(\widetilde{R})$, this implies that $\Phi(\widetilde{R})=[m-\delta, m+\delta]$ for some $\delta>\varepsilon$, violating Pareto optimality also in this case, since the convex hull of the support of $\lambda_{\widetilde{R}}$ is the interval $[a, m+\varepsilon]$.

So $\Phi$ cannot be Pareto optimal.

Thus, applying cabinet formation rules of the form $\Phi^{g}\left(g \in G^{\pi}, 0<\pi<1\right)$ may result in cabinets that (up to a set of voters of measure zero) contain the most left of most right voter of an election in their interior. In the last and main result of this paper we characterize a class of CFRs that do not suffer from this potential deficit, i.e., that are Pareto optimal. Note that, if $\pi \in\{0,1\}$, then $\Phi^{g}$ is Pareto optimal for every $g \in G^{\pi}$ by construction.

Let $J$ be an ordered index set. A sequence of cabinets $([a, b])_{j \in J}$ converges to a cabinet $[a, b]$ if $\left(a_{j}\right)_{j \in J}$ converges to $a$ and $\left(b_{j}\right)_{j \in J}$ converges to $b$. A CFR $\Phi$ is continuous if $\left(\Phi\left(R_{j}\right)\right)_{j \in J}$ converges to $\Phi(R)$ whenever $\left(R_{j}\right)_{j \in J}$ converges to $R$.

A CFR $\Phi$ is extreme strategy-proof if it is both $m^{0}$ - and $m^{1}$-strategy-proof. Hence, if a CFR is extreme strategy-proof, then no coalition can manipulate in such a way that the left endpoint or right endpoint of the cabinet moves closer to the ideal point of each of its members. In Remark 4.8 we further comment on this strategy-proofness condition.

We will characterize all CFRs that are anonymous, Pareto optimal, continuous, and extreme strategy-proof. To this end, let $H \subseteq G^{0} \times G^{1}$ be defined by

$$
H:=\left\{\left(h^{0}, h^{1}\right) \in G^{0} \times G^{1} \mid \max \left\{\alpha \mid h^{0}(\alpha)=0\right\}-\min \left\{\alpha \mid h^{1}(\alpha)=1\right\} \geq p\right\} .
$$

For a pair $h=\left(h^{0}, h^{1}\right) \in H$ we define the CFR $\Phi^{h}$ as follows. For a profile 


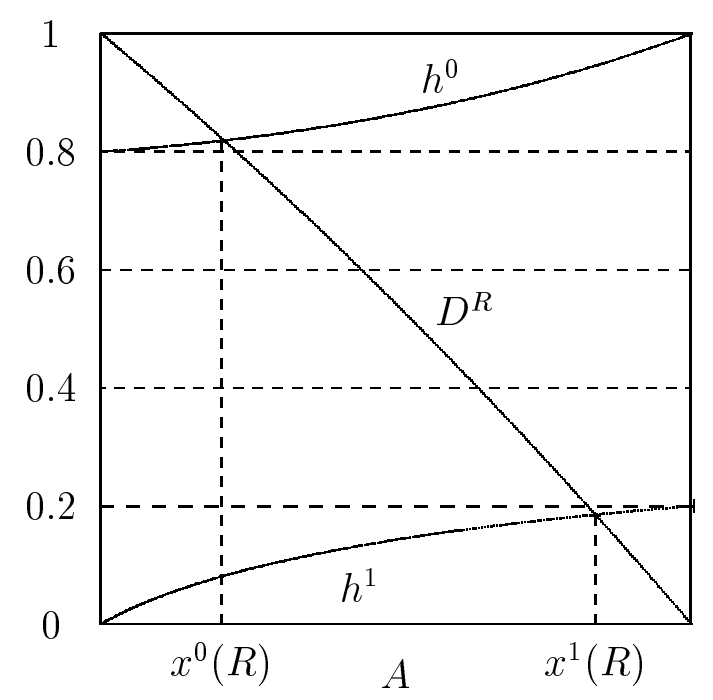

Figure 3: For this diagram, $p=0.6$. The distribution is $D^{R}$. The functions $h^{0}$ and $h^{1}$ have their domains on the vertical axis. The pair $h=\left(h^{0}, h^{1}\right)$ is in $H$. The resulting cabinet is $\Phi^{h}(R)=\left[x^{0}(R), x^{1}(R)\right]$.

$R \in \rho$ define, as before,

$$
x^{i}(R):=\max \left\{x \in A \mid h^{i}\left(D^{R}(x)\right) \geq x\right\}
$$

for $i=0,1$. Then $\Phi^{h}(R):=\left[x^{0}(R), x^{1}(R)\right]$. See Figure 3 for an illustration of this definition.

Theorem 4.6 A CFR $\Phi$ is continuous, Pareto optimal, extreme strategyproof, and anonymous, if and only if there is an $h \in H$ such that $\Phi=\Phi^{h}$.

Proof. Let $h \in H$. By definition, $\Phi^{h}$ is both $m^{0}$ - and $m^{1}$-continuous, and therefore continuous. Also, it is both $m^{0}$ - and $m^{1}$-Pareto optimal and therefore Pareto optimal. Anonymity is obvious, and extreme strategy-proofness follows from $m^{0}$ - and $m^{1}$-strategy-proofness. Let $R \in \rho$ be an arbitrary profile. We verify that $\lambda_{R}\left(\Phi^{h}(R)\right) \geq p$. Let $\alpha \in[0,1)$ with $h^{0}(\alpha)=0$. If $D^{R}\left(x^{0}(R)\right)<\alpha$, then $h^{0}\left(D^{R}\left(x^{0}(R)\right)\right) \leq h^{0}(\alpha)=0$, hence $x^{0}(R)=0$; however, $h^{0}\left(D^{R}(0)\right)=1$, a contradiction. Hence $D^{R}\left(x^{0}(R)\right) \geq \alpha$. Similarly, let $\alpha \in(0,1]$ with $h^{1}(\alpha)=1$. If $x^{1}(R)=1$ then $D^{R}\left(x^{1}(R)\right)^{+}=0 \leq \alpha$. If $x^{1}(R)<1$, consider $x \in A$ with $x>x^{1}(R)$. Then, by definition, 
$h^{1}\left(D^{R}(x)\right)<x$. Suppose $D^{R}(x)>\alpha$, then $h^{1}\left(D^{R}(x)\right) \geq h^{1}(\alpha)=1$, hence $x>1$, a contradiction. So $D^{R}(x) \leq \alpha$ for all $x>x^{1}(R)$ and hence $D^{R}\left(x^{1}(R)\right)^{+} \leq \alpha$. Therefore

$$
\begin{aligned}
\lambda_{R}\left(\Phi^{h}(R)\right) & =\lambda_{R}\left(\left[x^{0}(R), x^{1}(R)\right]\right) \\
& =D^{R}\left(x^{0}(R)\right)-D^{R}\left(x^{1}(R)\right)^{+} \\
& \geq \max \left\{\alpha \mid h^{0}(\alpha)=0\right\}-\min \left\{\alpha \mid h^{1}(\alpha)=1\right\} \\
& \geq p .
\end{aligned}
$$

For the converse, let $\Phi$ satisfy the conditions in the theorem. Since $\Phi$ is both $m^{0}$ - and $m^{1}$-continuous, Pareto optimal and strategy-proof, the existence of functions $h^{0} \in G^{0}$ and $h^{1} \in G^{1}$, satisfying, for every $R \in \rho$,

$$
\Phi(R)=\left[x^{0}(R), x^{1}(R)\right], x^{i}(R):=\max \left\{x \in A \mid h^{i}\left(D^{R}(x)\right) \geq x\right\} \text { for } i=0,1
$$

can be established in exactly the same way as in the proof of Proposition 4.4. The only thing left to show is that $h=\left(h^{0}, h^{1}\right) \in H$. Suppose not, i.e.,

$$
\max \left\{\alpha \mid h^{0}(\alpha)=0\right\}-\min \left\{\alpha \mid h^{1}(\alpha)=1\right\}<p .
$$

Denote

$$
\begin{aligned}
& \hat{\alpha}=\max \left\{\alpha \mid h^{0}(\alpha)=0\right\} \\
& \widetilde{\alpha}=\min \left\{\alpha \mid h^{1}(\alpha)=1\right\},
\end{aligned}
$$

then $p \leq \hat{\alpha}<1$ and $0<\widetilde{\alpha} \leq 1-p$, and $\hat{\alpha}-\widetilde{\alpha}<p$. Choose $\delta>0$ such that $2 \delta<p-\hat{\alpha}+\widetilde{\alpha}, \bar{\alpha}:=\hat{\alpha}+\delta<1$, and $\underline{\alpha}:=\widetilde{\alpha}-\delta>0$. Let $R \in \rho$ be a profile with a continuous decumulative distribution function satisfying $D^{R}\left(h^{0}(\bar{\alpha})\right)=\bar{\alpha}$ and $D^{R}\left(h^{1}(\underline{\alpha})\right)=\underline{\alpha}$. Then

$$
\begin{aligned}
\lambda_{R}\left(\Phi^{h}(R)\right) & =\lambda_{R}\left(\left[x^{0}(R), x^{1}(R)\right]\right) \\
& =D^{R}\left(h^{0}(\bar{\alpha})\right)-D^{R}\left(h^{1}(\underline{\alpha})\right) \\
& =\bar{\alpha}-\underline{\alpha} \\
& =\hat{\alpha}-\widetilde{\alpha}+2 \delta \\
& <p,
\end{aligned}
$$

a contradiction. Hence, $h=\left(h^{0}, h^{1}\right) \in H$.

We conclude this section with two remarks. 
Remark 4.7 The cabinet formation rules in Theorem 4.6 are not minimal but they can be chosen as 'minimal' as desired. More precisely, one can choose $h=\left(h^{0}, h^{1}\right) \in H$ such that

$$
\max \left\{\alpha \mid h^{0}(\alpha)=0\right\}-\min \left\{\alpha \mid h^{1}(\alpha)=1\right\}=p,
$$

and letting both $h^{0}$ and $h^{0}$ increase from 0 to 1 as fast as desired, say, both within an interval of length $\varepsilon$ with $\varepsilon>0$ as small as desired.

Remark 4.8 An extreme strategy-proof cabinet formation rule is easily seen also to be strategy-proof with respect to the following partial ordering on closed intervals or cabinets. If a voter's ideal point is to the left of the left end-point of the cabinet then any cabinet also to his right but with both endpoints (weakly) closer is preferred. Similarly with left and right reversed. If a voter's ideal point is inside a cabinet, then any cabinet with the voter's ideal point still inside but both end-points (weakly) closer is also preferred. This partial ordering is consistent with extensions of preferences from points to sets studied earlier in the literature, see Kannai and Peleg (1984), Barberà et al. (1984), Bossert (1989), Nehring and Puppe (1996), and Klaus and Storcken (2002).

While the first part of this description seems fairly natural, the last part is subject to discussion. Whether a cabinet that is smaller on both ends of the political spectrum but still contains one's favourite political party is preferable, may depend on the position of that party in the cabinet. If that party will occupy a more central position as a consequence of the decrease, then the answer may be affirmative, but otherwise that is less clear. On the other hand, it is unlikely that an ordering can be found that is uncontroversial on the one hand and not too sparse on the other hand.

\section{Concluding remarks}

An obvious objective for further research is to extend the results of this paper and in particular Theorem 4.6 on cabinet formation to more dimensions. Results for multi-issue voting in the finitely many agents framework (in particular Zhou, 1991) justify the conjecture that single-peakedness alone will not be sufficient to obtain possibility results for voting rules or cabinet formation rules. Further domain restrictions like separability of preferences (cf. Border and Jordan, 1983; Peters et al., 1992) may be required. 
Another interesting avenue for further research is to explore the potential role of simple games in a multi-dimensional cabinet formation framework with a continuum of agents. See Owen (1995, Chapter XVI) or Straffin (1994) for an overview of spatial games in the case with finitely many players.

\section{References}

Axelrod, R. (1970): Conflict of Interest: A Theory of Divergent Goals with Applications to Politics. Chicago: Markham.

Barberà, S., C.R. Barrett, and P.K. Pattanaik (1984): "On Some Axioms for Ranking Sets of Alternatives," Journal of Economic Theory, 33, 301-308.

Border, K.C., and J.S. Jordan (1983): "Straightforward Elections, Unanimity, and Phantom Voters," Review of Economic Studies, 50, 153-170.

Bossert, W. (1989): "On the Extension of Preferences over a Set to the Power Set: An Axiomatic Characterization of a Quasi-Ordering," Journal of Economic Theory, 49, 84-92.

Ching, S. (1997): "Strategy-Proofness and Median Voters," International Journal of Game Theory, 26, 473-490.

Ehlers, L., H. Peters, and T. Storcken (2002): "Strategy-Proof Probabilistic Decision Schemes for One-Dimensional Single-Peaked Preferences," Journal of Economic Theory, 105, 408-434.

Kannai, Y., and B. Peleg (1984): "A Note of the Extension of an Order to the Power Set," Journal of Economic Theory, 32, 172-175.

Klaus, B., and T. Storcken (2002): "Choice Correspondences for Public Goods," Social Choice and Welfare, 19, 127-154.

Moulin, H. (1980): "On Strategy-Proofness and Single-Peakedness," Public Choice, 35, 437-455. 
Nehring, K., and C. Puppe (1996): "Continuous Extensions of an Order on a Set to the Power Set," Journal of Economic Theory, 68, 456-479.

Owen, G. (1995): Game Theory. San Diego: Acadmic Press (third edition).

Peters, H., H. van der Stel, and T. Storcken (1992): "Pareto Optimality, Anonymity, and Strategy-Proofness in Location Problems," International Journal of Game Theory, 21, 221-235.

Riker, W.H. (1962): The Theory of Political Coalitions. New Haven / London: Yale University Press.

Straffin, P.D., Jr. (1994): "Power and Stability in Politics," in: Handbook of Game Theory with Economic Applications, Vol. 2, edited by R.J. Aumann and S. Hart. Amsterdam: North-Holland.

De Swaan, A. (1973): Coalition Theories and Cabinet Formations. Amsterdam: Elsevier Scientific Publishing Company.

De Vries, W.M. (1999): Governing with Your Closest Neighbour: An Assessment of Spatial Coalition Formation Theories. Ph.D.-thesis, University of Nijmegen, The Netherlands.

Zhou, L. (1991): "Impossibility of Strategyproof Mechanisms in Economies with Pure Public Goods," Review of Economic Studies, 58, 107-119. 